\section{La constitución}

\section{de las Culturas}

Pedagógicas

Modernas:

\section{Una aproximación conceptual}

\section{The constitution of Modern \\ Pedagogic Cultures: \\ A conceptual approach}

\author{
Fundação das culturas \\ pedagógicas modernas: \\ Uma abordagem conceitual
}

\section{Carlos Ernesto Noguera Ramírez}

* Profesor Asociado de la Universidad Pedagógica Nacional, Bogotá, Colombia. Doutor em Educação de La Universidade Federal do Rio Grande do Sul, RS, Brasil. Investigador del Grupo Historia de la Práctica Pedagógica en Colombia.

Correo electrónico: c.guera@hotmail.com

\section{Resumen}

El artículo parte de la tesis según la cual la constitución de la llamada "Pedagogía Moderna" corresponde en realidad a la instauración, durante el siglo XIX, de tres tradiciones intelectuales en cuyo centro es posible identificar problemáticas o conceptos particulares propios de sus ambientes culturales: el francófono, el germánico y el anglosajón. Así, las aproximaciones a la educación para la tradición francófona, a la Building o formación para la tradición germánica y al currículo para la tradición anglosajona, quedan esbozadas aquí a manera de una 'cartografía conceptual' de lo que podría llamarse las Culturas Pedagógicas Modernas.

\section{Palabras clave}

Culturas pedagógicas, ciencias de la educación, currículo, formación, pedagogía moderna.

\section{Abstract}

This article starts through the thesis according to which the constitution of the called "Modern Pedagogy" really belongs to the establishment, during XIX century, of three intellectual traditions in which focus it is possible to identify problems or particular concepts proper of their cultural environments: the Francophone one, the Germanic one, and the Anglo-Saxon one. In this way, the approaches to education to the Francophone tradition, to the Building or Training for the Germanic tradition, and to the Curriculum for the Anglo-Saxon tradition, are outlined here like a kind of "Conceptual Cartography" of what could be named "Modern Pedagogic Cultures".

\section{Key words}

Pedagogic cultures, sciences of education, curriculum, training, modern pedagogy.

\section{Resumo}

0 artigo estabelece que a constituição da "pedagogia moderna" corresponde à instauração, no século XIX, de três tradições intelectuais nas que se identificam problemáticas ou conceitos específicos próprios de seus ambientes culturais: francófono, germânico e anglo-saxão. Por conseguinte, as abordagens à educação para a tradição francófona, à Bildung o formação para a tradição germânica e ao currículo para tradição anglo-saxônica esboçam-se à maneira de uma 'cartografia conceitual' do que pode denominar-se culturas pedagógicas modernas.

\section{Palavras-chave}

Culturas pedagógicas, ciências da educação, currículo, formação, pedagogia moderna.

Fecha de recepción: 26 de julio de 2010 Fecha de aprobación: 16 de noviembre de 2010

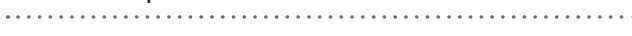

Pedagogía y Saberes No.33. Universidad Pedagógica Nacional. Facultad de Educación. 2010, pp. 9 - 25 
l presente artículo corresponde a una investigación más amplia sobre la constitución del discurso pedagógico moderno (Noguera, 2009), y parte de una tesis según la cual la constitución de la llamada "Pedagogía Moderna" corresponde en realidad a la instauración, durante el siglo XIX, de tres tradiciones intelectuales en cuyo centro es posible identificar conceptos y problemáticas particulares, propias de tres ambientes culturales: el anglosajón, el francófono y el germánico. De manera muy general -y tal vez muy esquemática-, se podría caracterizar cada una de las culturas pedagógicas1 como sigue: en el corazón de la cultura pedagógica angloamericana está la preocupación por el currículo como forma de organización de los contenidos y de las actividades de enseñanza y aprendizaje; en el centro de la cultura pedagógica germánica está el problema de la Bildung o formación (diferente de la educación o de la enseñanza) y, a partir de ella, la diferenciación entre pedagogía (interesada en la educación en general) y didáctica (interesada en la formación y en los procesos de enseñar y aprender); por último, como distintivo de la cultura pedagógica francófona, se encuentran las elaboraciones acerca del concepto de educación y, con ellas, el establecimiento de las condiciones de posibilidad que llevaron, posteriormente, entre finales del siglo XIX e inicios del XX, a la constitución de, por lo menos, dos "ciencias de la educación" - la sociología de la educación y la psicopedagogía- como formas particulares de abordar la amplia problemática representada por ese nuevo concepto.

A pesar de esas marcas particulares, cada cultura pedagógica no puede ser considerada como independiente y funcionando aisladamente de las otras. Efectivamente, ellas se interceptan produciendo significativos intercambios; por ejemplo, como nos muestran las historias de la pedagogía, el movimiento herbartiano tuvo gran influencia, tanto en los países francófonos como en los Estados Unidos de América, y la diferencia entre educación e instrucción, genera-

1 Utilizo aquí el concepto de cultura pedagógica que Zuluaga y Herrera (2009, p. 27) definen como: "la constitución de saberes donde se despliegan prácticas; los sistemas de elaboración simbólica; los sistemas de representación e intercambio; y la generación de objetos y dispositivos pedagógicos, producidos en una sociedad o conjunto de sociedades, a propósito de la educación" Una cultura pedagógica así entendida, abarca "la educación formal, la no formal y la informal, pero cada una de diferente modo. Así mismo, comprende: las conceptualizaciones internas a sus propias dimensiones, el abanico de saberes y disciplinas que la integran; así como la organización de la intelectualidad en torno a ellas, y los modos de conducta de los sujetos comprometidos en las prácticas discursivas como son: la intelectualidad, los profesores, los maestros, los alumnos y los funcionarios públicos, que interactúan en las prácticas" (p. 28). da en los debates de los revolucionarios franceses, fue asimilada por todas las tradiciones. Por su parte, existieron -y aún existen- versiones angloamericanas y germánicas de una sociología o de una psicología de la educación.

“...en el corazón de la cultura pedagógica angloamericana está la preocupación por el currículo como forma de organización de los contenidos y de las actividades de enseñanza y aprendizaje; en el centro de la cultura pedagógica germánica está el problema de la Bildung o formación... por último, como distintivo de la cultura pedagógica francófona, se encuentran las elaboraciones acerca del concepto de educación..."

Estudios más o menos recientes han destacado el hecho de que las culturas pedagógicas obedecen a particulares condiciones sociales, culturales, políticas y económicas (Hopmann \& Riquarts, 2000; Westbury, Hopmann \& Riquarts 2000). La teoría curricular, por ejemplo, pudo ser producida en el marco de los problemas planteados por la cuestión de la creación y administración eficiente de un sistema de instrucción elemental de masas, en una sociedad urbanizada, industrializada y descentralizada como fue el caso de los Estados Unidos de América durante el siglo XIX. Por el contrario, la consolidación de la Didaktik sólo fue posible en el contexto de un mundo de pequeñas ciudades poco industrializadas y un sistema escolar centralizado, como el de la Prusia del siglo XIX. Pero, a pesar de esas diferencias particulares, existió un elemento común íntimamente ligado a la constitución de las tres tradiciones: la creación, en los diferentes países y con ritmos distintos y características propias, de aparatos gubernamentales encargados de la administración e inspección de la llamada "instrucción pública". Se trata de la expansión de la escolarización en la población, pero ya no como un asunto de interés de las comunidades religiosas o de la Iglesia sino, claramente, como un asunto cada vez más reivindicado por el Estado como de su potestad. Dilthey (1968) analizó ese proceso de estatalización de la instrucción pública como la constitución de un "Estado Educador" cuya forma primera fue Prusia².

2 Sobre las particularidades del precoz proceso de expansión de la escuela pública en Prusia y en Austria, ver Melton (2002) 
Una caracterización a fondo de cada una de las culturas pedagógicas implica un trabajo que desborda las posibilidades de este ensayo y requeriría de una investigación mucho más amplia sobre la base de un grupo internacional de investigadores. Lo que aquí intento hacer es una aproximación conceptual, el esbozo de una "cartografía conceptual"; sin embargo, la intención no es llevar a cabo una caracterización completa de los desarrollos de cada una de esas culturas pedagógicas; no se trata de una cartografía conceptual de la producción de los diferentes autores o representantes de cada cultura pedagógica. Se trata de un trabajo más modesto, aunque central en nuestras discusiones pedagógicas contemporáneas: diseñar un mapa general a partir del análisis de un concepto o problemática central en cada una de las tradiciones, con el propósito de destacar la fertilidad del campo del saber pedagógico moderno, su pluralidad y productividad, diluida en la idea de la existencia — construida por la historiografía pedagógica- de una supuesta "pedagogía moderna".

\section{La emergencia del concepto moderno de "educación" como condición de posibilidad para una Ciencia de la Educación}

\author{
"Mi asunto era totalmente nuevo después del libro \\ de Locke, y temo mucho que continúe siéndolo \\ después del mío"
}

(Rousseau, 1999, p. 4)

Contrario a lo que podría pensarse, el concepto de educación es relativamente reciente en el lenguaje del saber pedagógico. Tuvo su emergencia a fines del siglo XVII y su delimitación aconteció en los siglos XVIII y XIX. Varios autores corroboran esta aseveración. René Hubert, en su Tratado de Pedagogía General, afirma lo siguiente:

Según el Diccionario general de Hatzfeld, Daimesteter e Thomas, [el término éducation] no lo encontramos en la lengua francesa antes de 1527. Está en todos los léxicos a partir de de 1549, así como en el Diccionario francés-latino de Robert Estienne $^{3}$, donde se le relaciona con la alimentación. Pero todavía no aparece más que raramente en los textos. Por lo demás, etimológicamente no

3 Educatio: Nourrissier. Educatrix. Educatrix, pen. prod. Verbale foem. gen. Colum. Nourrisse. Educatio, Verbale. Cic. Nourriture, Education. EDUCO educis, pen. prod. eduxi, eductum, educere. Tirer ou Mener hors. Colores educere. Catul. Produire fleurs de diverses couleurs. Educere cirneam vini. Plaut. Tirer hors $\|$ (Estienne, 1552, p. 453). es más que una transcripción del latín, debido a los humanistas del Renacimiento. El latín empleaba la palabra correspondiente indiferentemente para el cultivo de las plantas, el cuidado de los animales, la nutrición y la institución de los seres humanos. En 1649, la traducción francesa no conoce todavía más que la primera acepción de esta palabra. Sólo entiende la educación como la formación del espíritu y del cuerpo, y la hace consistir en la instrucción ${ }^{4}$ : «el cuidado que se tiene de la instrucción de los niños, sea en lo que se refiere a los ejercicios del espíritu, sea en lo que se refiere a los ejercicios del cuerpo» (Hubert, 1952, pp. 13-14).

Para Compayré (1897), la palabra éducation es relativamente nueva en lengua francesa y tal vez haya sido Montaigne quien la utilizó por primera vez en sus Ensayos, aunque habitualmente prefería la expresión institution des enfants de donde procede la palabra instituteur (institutor). Los escritores del siglo XVI decían nourriture (alimentación, nutrición) y sólo después del siglo XVII el término éducation ingresó en el lenguaje corriente para designar el arte de erigir (elevar, mejorar, perfeccionar) a los hombres. También señala Compayré que la educación es propia del hombre y, por lo tanto, conviene reservar sólo al hombre esa "bella palabra" (Compayré, 1897, p. $10)$, pues los animales se adiestran y las plantas se cultivan: "Sólo el hombre es susceptible de educación porque sólo él es apto para gobernarse a sí mismo, para devenir una persona moral" (p. 10).

Según Corominas (1954), en castellano la palabra educación data del siglo XVII, pues "hasta entonces se había dicho siempre criar, que es la voz que emplea aún Cipr. [Cipriano] de Valera en su traducción de la Biblia, a finales del siglo XVI, allí donde Scio, siglos antes, escribió educar" (p. 216). Cunha (1986), también señala el siglo XVII como el momento de aparición de la palabra educação en la lengua portuguesa. Sin embargo, tal vez fuese Some thoughts concerning education (1693), de John Locke - escrita en un idioma vernáculo y no en latín-, el texto donde se inauguró el término education y, más importante aún, donde se establecieron sus fundamentos. Pero fue Rousseau, setenta años después, quién delimitó el término en su sentido propiamente moderno.

La frase con la que abre esta sección pertenece al prefacio del Emilio, donde parece expresar la certeza que tenía Rousseau sobre la novedad del problema que

4 Education.s. f. Le soin qu'on prend de l'instruction des enfants, soit en ce qui regarde les exercices de l'esprit, soit en ce qui regarde les exercices du corps. Bonne education. mauvaise education. l'education des enfants. prendre soin de l'education des enfants. il se sent bien de la bonne education qu'il a euё. EFF||. (Académie Française, 1694). 
estaba formulando, sobre su carácter extemporáneo. Aunque Locke emplease por primera vez aquel término - education - siete décadas antes en sus conocidos Pensamientos sobre la educación, es a partir del Emilio que esa palabra adquiere el significado moderno; de ahí que, en sentido estricto - y contrariamente a la afirmación de Narodowski $(1995,2001)$-, podamos decir que es el Emilio y no la Didáctica Magna la obra fundadora de la 'pedagogía moderna', o mejor aún, de las culturas pedagógicas modernas. Coincido con $\mathrm{Na}$ rodowski en el carácter transdiscursivo de la didáctica comeniana ${ }^{5}$, pero, siguiendo su perspectiva, se diría que con el Emilio se constituye en otro 'paradigma transdiscursivo' a partir del cual, y mediante la incorporación de la tradición didáctica, se desarrollaron las tres culturas pedagógicas modernas. Sin duda, con el trabajo de Comenio queda fundada la didácticapero es a partir de las elaboraciones de autores de la tradición intelectual germánica, en los inicios del siglo XIX, que se integrará dentro de la Pädagogik o Ciencia de la Educación ${ }^{6}$. De esa forma, la educación y sus desarrollos - y ya no sólo la enseñanza - estuvieron en el centro de los discursos pedagógicos.

Retomando las palabras de Rousseau transcritas al inicio de esta sección, debemos aceptar que tenía razón cuando temía que su asunto iba a permanecer como novedad aún después de su libro, pues sus elaboraciones sólo serán plenamente desarrolladas al final del siglo XIX a partir de la psicopedagogía francófona y la psicología del aprendizaje anglosajona. En otras palabras, podría decirse que el Emilio es el esbozo de un nuevo "régimen de veridicción"7

5 Sobre este aspecto afirma Narodowski: "De hecho, Comenio está implantando un paradigma transdiscrusivo, que será en núcleo de hierro del discurso pedagógico moderno. Esa transdiscursividad se refiere al hecho de que el núcleo común instalado por la obra comeniana será compartido - pese a sus diferencias teóricas e ideológicas- por los pedagogos y pedagogías de la Modernidad" (2001, p. 16).

6 En particular, desde Herbart, con su propuesta de una "educación a través de la enseñanza" - y a pesar de que posteriores propuestas hayan considerado la posibilidad de hablar de una Didáctica o Teoría de la Formación humana en lugar de "pedagogía" (ver, por ejemplo, Willmann, 1948), se inició una recomposición de la didáctica clásica que, sobre la base del concepto de Bildung, amplió el problema de la erudición o instrucción (eruditio) en la dirección de la educación (institutio). Hay que decir, sin embargo, que la didáctica continuó sus desarrollos particulares como disciplina acerca de los problemas de la enseñanza y el aprendizaje constituyéndose, por ejemplo, una Didáctica General (allgemeine Didaktik) y otras específicas (Fachdidaktik).

7 "[...] el régimen de veridicción, en efecto, no es una ley determinada de la verdad, [sino] el conjunto de las reglas que permiten, con respecto a un discurso dado, establecer cuáles son los enunciados que podrían caracterizarse en él como verdaderos o falsos" (Foucault, 2007, p. 53). en el campo del saber pedagógico, campo que sólo será plenamente actualizado y desarrollado un siglo después. Es importante aclarar que la constitución de un nuevo 'régimen de veridicción' no es asunto de un autor o una obra en particular, puesto que implica un proceso anónimo que involucra (que crea) autores y obras funcionando como sus superficies de emergencia y desarrollo. El Emilio es esa primera superficie de emergencia de los nuevos enunciados o reglas de verdad para el discurso pedagógico moderno liberal, discurso que sólo hasta inicios del siglo XX funcionará como un 'régimen de veridicción' propiamente dicho.

En el inicio de su Emilio, Rousseau, citando a Varrón, señala que la palabra educación antiguamente significaba alimento y estaba relacionada con la idea de crianza: educit obstetrix, educat nutrix, instituit paedagogus, docet magister, frase que, siguiendo la línea de argumentación aquí desarrollada, debería ser traducida como: "Trae a luz la partera, cría la nodriza, educa el preceptor, enseña el maestro". El término educere significa sacar, extraer; educare significa alimentar, criar; a su vez instituere significa formar, fundar, establecer y, docere, puede ser traducido por enseñar o instruir. De esta forma se aclara la diferencia que pretende establecer Rousseau a propósito de los tres tipos de actividad realizados por la nodriza (crianza), el ayo (institución o educación en sentido moderno) y el maestro (instrucción o enseñanza). Esta afirmación se justifica con la mención del propio Rousseau sobre la confusión existente en su época entre esas tres actividades, y su aclaración sobre la necesidad de un solo guía o conductor para el niño, guía que debe ser el gouverneur ${ }^{8}$ (ayo): "yo más bien llamaré ayo [gouverneur] que preceptor al maestro de esta ciencia, porque no tanto es su oficio instruir como conducir. No debe dar preceptos, debe hacer que los halle el alumno" (p. 15). La educación es más dirección y conducción que instrucción o enseñanza y, en ese sentido, el concepto está más próximo de las ideas de Locke (y, en esa línea, de la institutio $^{9}$ de los humanistas) que de la eruditio de la didáctica, pues para formar al 'hombre' no es

8 Esta palabra francesa que literalmente podría ser traducida como "gobernador", expresa mejor la idea de Rousseau. En español, la palabra ayo significa "Persona encargada en las casas principales de custodiar niños o jóvenes y de cuidar de su crianza y educación" y preceptor es la "persona que enseña" Real Academia Española, 1992.

9 Durante el Renacimiento, humanistas como Erasmo, Vives y Montaigne utilizaron el término latino institutio para referirse a la necesidad de una "educación" o "formación" en perspectiva moral, centrada en la constitución de prácticas y hábitos para el ejercicio de la virtud, por oposición a la vía escolástica y didáctica, empeñada en procurar una erudición-eruditiopara lo cual privilegiaba la enseñanza y la instrucción. Al respecto ver Noguera (2009). 
necesaria una enseñanza de las ciencias. En varias oportunidades a lo largo de su libro Rousseau (1984) aclara este punto:

No se trata de enseñarle las ciencias, sino de inspirarle la afición por ellas, y darle métodos para que las aprenda cuando se desenvuelva mejor su afición. He aquí ciertamente el principio fundamental de toda buena educación (p. 118).

Considerad lo primero, que rara vez debéis proponerle lo que él ha de aprender; a él le toca desearlo, indagarlo, hallarlo; a vos ponerlo a su alcance, hacer con su maña que nazca ese deseo, y darle medios para que le satisfaga (p. 125).

Tiene [Emilio] un espíritu universal, no por la luces sino por la facultad de adquirirlas; un espíritu despejado, inteligente, apto para todo, y como dice Montaigne, si no instruido, instructible. Bástame con que sepa hallar el para qué sirve en todo cuanto haga, y el por qué en todo cuanto crea: porque repito que no es mi objeto darle ciencia, sino enseñarle a que la adquiera cuando la necesite [...] (p. 150).

Sin embargo, y a pesar de su aproximación a las ideas del filósofo inglés, la educación de Rousseau inaugura un nuevo momento para el saber pedagógico. Locke, con su énfasis en la disciplina del entendimiento, en la constitución de hábitos, en la importancia del ejercicio, en la repetición, se inscribe aún en el marco de lo que podríamos calificar como una forma de gobierno pedagógico disciplinar, mientras que Rousseau —con su novedosa idea de educacióninaugura la era de un "gobierno pedagógico liberal", es decir, establece una nueva forma de educación, conducción, dirección (gobernamiento) del 'hombre', fundamentada en las ideas de naturaleza, libertad e interés (o deseo) del agente que aprende (el niño) en un 'medio' especialmente adaptado para tal fin (medio que ya no es la casa paterna, como en el caso del gentleman de Locke, ni de la escuela, como en el caso de Comenio) ${ }^{10}$. Pero ¿en qué consiste la novedad de esa éducation rousseauniana?

Se trata de un concepto que aparecerá íntimamente relacionado con otros conceptos claves dentro de lo que Foucault (2006-2007) denominó la nueva "gubernamentalidad liberal”. La educación natural consiste en abrir paso a la espontaneidad, permitir que la mecánica interna del individuo actúe libremente, un dejar hacer, es decir, dejar de intervenir como medida para permitir que la naturaleza opere. Tenemos,

10 Sobre las relaciones entre el concepto de gubernamentalidadacuñado por Foucault $(2006,2007)$ en sus cursos de 1978 y 1979- y el análisis de la constitución del discurso pedagógico moderno ver Noguera (2010). entonces, libertad y naturaleza como los conceptos más generales y, ligados a ellos, los de interés, crecimiento, desarrollo, maduración y medio: conceptos del nuevo lenguaje que, desde entonces hasta hoy, estarán en el corazón de los discursos pedagógicos. En este sentido, la nueva razón educativa que funda Rousseau precisó de libertad: sólo podía funcionar si existiese libertad, necesitaba de ella y, por tanto, tenía que producirla. Pero también necesitó de una "naturaleza" propia del sujeto, de ahí que el Emilio no fue el esbozo o el 'descubrimiento' de las leyes naturales del desarrollo del niño - como creía Claparède ${ }^{11}$-, sino que más bien podría analizarse como la superficie de emergencia de una nueva gramática que orientará el discurso pedagógico o, también, de un conjunto de reglas a partir de las cuales se construyó el discurso pedagógico considerado moderno.

Sin embargo, esta nueva gramática no comenzó a funcionar con la publicación del Emilio- hecho que el propio Rousseau advirtiera en su prólogo, como ya vimos - sino que su implementación tuvo que esperar algo más de un siglo a la constitución de la psicopedagogía francófona (Binet, Claparède, Bovet) y la psicología del aprendizaje anglosajona (Thorndike). Entretanto, hubo en Francia dos importantes tentativas de fundación de una ciencia de la educación: la primera, denominada 'pedagogía filosófica' o 'filosofía pedagógica' (Dubois, 2007), cuyo principal representante fue Gabriel Compayré (1843-1913) y la segunda, la sociología de la educación, impulsada por Emile Durkheim (1858-1917).

En su Curso de Pedagogía (1897), señalaba Compayré: "Nadie pone en duda actualmente la posibilidad de una ciencia de la educación" (Compayré, 1920, p. 10); pero consideraba pertinente resaltar el error común de confundir pedagogía con educación: "La pedagogía es, por decir así, la teoría de la educación y la educación la práctica de la pedagogía. Así como se puede ser retórico sin ser orador, se puede ser pedagogo, es decir, conocer a fondo las reglas de la educación, sin ser educador, o sea, sin poseer la habilidad de educar prácticamente a los niños" (Compayré, 1920, p. 11). También — retomando las discusiones de los revolucionarios, particularmente de Condorcet (Compayré escribió un libro sobre él)-, coincidía en diferenciar entre educación e instrucción: "El uso general consiste aún en reservar la palabra educación

11 En su libro La educación funcional se incluye un artículo titulado "J.J. Rousseau y la concepción funcional de la infancia", en el cual muestra que el filósofo había expresado claramente en su Emilio las cinco leyes de la concepción funcional de la infancia: ley de la sucesión genética, ley del ejercicio genéticofuncional, ley de la adaptación o de utilidad funcional, ley de la autonomía funcional, y ley de la individualidad. Ver: Claparède (2007). 
para señalar la formación de las costumbres y del carácter. El objeto de la educación propiamente dicha, como distinta de la instrucción, es precisamente la cultura de la voluntad y del corazón por oposición a la de la inteligencia" (p. 53; cursivas del autor).

Esa diferenciación tiene implicaciones para la teoría pedagógica, pues establece para ella dos puntos de partida: el sujeto pensante que se procura educar y los objetos que se deben enseñar:

En el primer caso se parte de la naturaleza del hombre, se consideran las leyes de la formación de las facultades y se proponen los métodos generales de cultura conforme con esas leyes. En el segundo se parte de cada una de las diversas ramas de la enseñanza, se determina su naturaleza y sus caracteres y se establecen métodos de instrucción en conformidad con esos caracteres.

Hay, en otros términos, métodos de cultura, deducidos de las leyes de la psicología, y métodos de instrucción que, sin dejar de estar de acuerdo con la psicología, se inspiran sobretodo en la naturaleza de los conocimientos que se han de enseñar (Compayré, 1920, p. 57).

Así, existe una pedagogía teórica y una pedagogía práctica: la primera vinculada con el sujeto de la educación que es el niño; la segunda, con el objeto de la educación que son los métodos de enseñanza y las reglas del régimen escolar. Con esta división en la pedagogía, Compayré retoma las elaboraciones tanto de la didáctica germánica como de la 'metodología' belga y suiza:

El estudio de los métodos de enseñanza constituye una de las divisiones más importantes de la ciencia pedagógica. Para darle un nombre los pedagogos de algunos países han tomado de la filosofía el nombre de metodología. Otros han llamado didáctica al arte de enseñar, y M. Daguet, en su manual de pedagogía, aventura el nombre de metódica (p. 249).

Aquí deja claro el autor que no se trata del establecimiento de un método, el método de enseñanza (como era el caso de la antigua didáctica comeniana o aún de la pestalozziana), sino de diversos métodos que varían según la naturaleza de la enseñanza o de la materia a ser enseñada. Sobre este aspecto, señala que los profesores en Bélgica y Suiza distinguen una 'metodología general', que trata de los principios comunes a todo método, de una 'metodología especial' que "examina sucesivamente las diversas materias de enseñanza y procura los mejores medios que se han de utilizar en cada ciencia y en cada estudio" (p. 249). No obstante, señala el peligro de abusar en el estudio de los métodos que, en algunos casos, llegan casi a constituir una nueva escolástica llena de fórmulas eruditas, divisiones sutiles y términos pedantes:

Se abren esos manuales de pedagogía que están de moda en Bélgica y en Alemania y se encuentran interminables páginas consagradas a la distinción de los principios, de los modos, de los procedimientos y de los métodos de enseñanza; catálogos nutridos que enumeran nada menos que ocho formas de enseñanza, la forma acromática o de exposición ininterrumpida, la erotemática o exposición interrumpida, que comprende otras siete fórmulas distintas, la catequética, la socrática, la heurística, la repetitoria, la examinatoria, la analítica y sintética y la paralógica, todo sin prejuicio de la distinciones de los métodos y de los procedimientos en, intuitivo, comparativo, de oposición, etimológico, de razonamiento, descriptivo, de observación, repetitorio, sinóptico, de reproducción, ¡y once más aún! (Compayré, 1920, p. 252; cursivas del autor).

Frente a estos 'abusos' establece tres principios generales a los cuales se debe adaptar cualquier método: 1) Las características propias de los conocimientos que se van a enseñar, 2) Las leyes de la evolución mental en las diversas edades de la vida y, 3) El objeto propio y la extensión de cada grado de instrucción. Esos principios y la división de la pedagogía en teórica y práctica, así como la aclaración del 'sujeto' y del 'objeto' de la ciencia de la educación, son las principales contribuciones de esta primera tendencia. Queda sólo por analizar -de manera breve-, su concepción de educación y disciplina para percibir su localización en ese período intermedio entre lo que he denominado el gobierno (gobernamiento) pedagógico disciplinar y el gobierno pedagógico liberal.

Después de un balance de las definiciones de educación dadas por diferentes autores franceses, ingleses y alemanes, Compayré propone en su Curso ésta fórmula: "La educación es el conjunto de los actos reflexivos por medio de los cuales se ayuda a la naturaleza en el desarrollo de las facultades físicas, intelectuales y morales del hombre, para buscar su perfección, su felicidad y la realización de su destino social" (p. 18). Se trata de una educación, que él mismo llama 'liberal', en cuanto prepara para el libre desarrollo de la razón; tal educación liberal no aspira a una 'alta instrucción intelectual', pues es suficiente una formación elemental, siempre que abra la inteligencia y fortifique la energía moral.

Coincide con Rousseau en que tal educación debe estar en conformidad con las leyes de la naturaleza, pero considera que aquello que se llama naturaleza, en el fondo, es un ideal que cada pedagogo concibe a su manera y que, como señala el pensador inglés 
Alexander Bain, existen en la naturaleza humana instintos malos como la cólera, el odio, la antipatía, la envidia, entre otros. Solo se puede abandonar la naturaleza a sí misma y, por el contrario, es necesario establecer unas restricciones, como propone Kant. También, coincide con Rousseau en que la educación es producto de la libertad, pues el hombre no es un ser inerte y pasivo, sino libre y activo: "el espíritu no es una materia inerte que se deja formar a voluntad y obedece pasivamente a todo aquello que se hace en ella, como el mármol o la madera al cincel del artista. Lejos de eso, el espíritu del niño reacciona sin parar y mezcla su acción propia a la del educador" (Compayré, 1920, p. 23). Pero, evidentemente, no es una colaboración equivalente: la actividad del alumno debe estar comprometida con su educación; por lo tanto, debe estar al servicio de la acción educativa del profesor, colaborar con él para llegar hasta donde se le conduce. De ahí que:

La educación no abandona la naturaleza a sí misma sino que la vigila, le dicta sus reglas y, en caso de necesidad, la reprime. De un modo general, es obra de la autoridad al igual que la libertad, pues la autoridad adquirida por el maestro que sabe hacerse estimar y obedecer, le permitirá acudir al convencimiento con más frecuencia que a la represión. Cuanto más autoridad tenga, menos necesitará usarla (p. 24).

Pero, no se debe olvidar que el fin último de la educación es el cultivo del carácter; por eso, no se debe temer a la libertad, sino encontrar en el propio alumno el freno necesario para reformar las pasiones y los malos instintos, es decir, buscar con la educación el establecimiento de mecanismos para que el propio sujeto se gobierne a sí mismo. En esta perspectiva, Compayré considera, en la vía de Herbart, que la disciplina tiene un fin superior que es la formación del carácter, motivo por el cual resulta central para la educación. Así, antes que basarse en un conjunto de premios y castigos, debe ser preventiva, y eso sólo es posible si el profesor sigue un método adecuado, una regularidad y continuidad de los ejercicios escolares, una utilización correcta del tiempo, una clasificación de los discípulos (no sólo por su edad, sino por su grado de instrucción y desarrollo intelectual) y una vigilancia rigurosa:

Aún así, las reglas no bastan. El discípulo no es aún lo bastante dueño de sí mismo ni lo bastante enérgico y bien intencionado para seguir espontáneamente la marcha que traza el reglamento. Hay que contar con los desfallecimientos de la voluntad, con el aturdimiento de la infancia, con la disipación, con la pereza y con el mal deseo. A la mirada vigilante del maestro corresponde asegurar la práctica de las leyes escolares. La disciplina es más fácil con un maestro activo que vigila todos los movimientos, que está al acecho de las disposiciones, que corta con una palabra o con una mirada una conversación que comienza, que reanima la atención en el momento en que se adormece, y que, en una palabra, está siempre presente en las cuatro esquinas de la escuela y es, por decirlo así, el alma del salón (p. 434).

“...Compayré considera, en la vía de Herbart, que la disciplina tiene un fin superior que es la formación del carácter, motivo por el cual resulta central para la educación. Así, antes que basarse en un conjunto de premios y castigos, debe ser preventiva..."

Ahora bien, esa intensa y permanente vigilancia no se puede detener en las puertas de la escuela: un buen profesor debe averiguar lo que hacen los niños en el seno de la familia y hasta cómo se comportan en la calle y los caminos, y para eso debe establecer una estrecha alianza con los padres informándolos periódicamente del progreso, del trabajo y de los defectos de los niños. Sin embargo, debe recordar que el fin de la disciplina es volverse inútil; aunque sea necesaria una sujeción, ella no impide la libertad "que es la disciplina que nos imponemos a nosotros mismos, y el fin de la educación en todos los grados es hacer hombres libres" (p. 441). Citando a M. Gréard, concluye Compayré:

[...] substituir insensiblemente a las reglas que se le han dado las que él mismo se dé, a la disciplina de afuera aquella de adentro; liberarlo, no de un solo golpe al modo antiguo, sino día por día, rompiendo a cada progreso un eslabón de la cadena que ataba su razón a la razón del otro; enseñarle a salir de sí mismo, a juzgarse, a gobernarse como juzgaría y gobernaría a los otros; mostrarle, en fin, las ideas del deber público y privado que imponen a su condición humana y social: tales son los principios de la educación que de la disciplina escolar hace pasar al niño a la disciplina de su propia razón y crea, al ejercitarla, su personalidad moral (Compayré, 1920, p. 442).

Queda claro, hasta aquí, cómo en esa primera tendencia de la 'ciencia de la educación' se va construyendo una nueva 'educación liberal', sin por ello abandonar los anteriores fundamentos disciplinares. 
El paso hacia un gobierno (gobernamiento) pedagógico liberal no significó una ruptura con la anterior tradición, sino una readecuación, una reacomodación cuyo resultado fue la producción de nuevos conceptos y prácticas.

En una perspectiva distinta de Compayré, Emile Durkheim, profesor del curso de pedagogía en la Facultad de Artes de Bordeaux entre 1887 y 1902 -año en que asumió el cargo de catedrático sustituto de "Ciencias de la Educación" en la Sorbona-, llamaba la atención sobre el hecho de que los pedagogos modernos estuviesen de acuerdo, casi en su totalidad, en ver la educación como un asunto eminentemente individual. Por el contrario, decía él: "Considero como el postulado mismo de toda especulación pedagógica que la educación es un ente eminentemente social, tanto en sus orígenes como por sus funciones, y que, por tanto, la pedagogía depende de la sociología más estrechamente que de cualquier otra ciencia" (Durkheim, 2003, p. 115).

Con esta observación, Durkheim abría una nueva perspectiva en la comprensión de la educación, al tiempo que inauguraba un nuevo intento de someter a la pedagogía a los dictámenes de una disciplina externa. Si los filósofos y psicólogos parecían aliados en la tentativa de considerar a la pedagogía como una ciencia aplicada derivada de las psicología, ahora Durkheim reclamaba para la incipiente ciencia social el derecho de subordinar la pedagogía, dado el carácter eminentemente social que concedía a la educación. Pero Durkheim fue más allá y, aunque reconociese como sus colegas filósofos que la pedagogía era una teoría práctica, establecía una clara diferencia entre ésta y una Ciencia de la Educación. No dudaba que, según los nuevos cánones de cientificidad de su época, era posible que la educación fuera un objeto de estudio científico, pero la pedagogía, en cambio, no sería esa ciencia cuyo objeto era la educación. En la segunda lección de su curso La educación moral, el sociólogo decía: "Ahora bien, si la pedagogía no es una ciencia, tampoco es un arte [...] La pedagogía es, por tanto, algo intermedio entre el arte y la ciencia" (Durkheim, 2002, p. 37).

Tal diferenciación obedeció, tanto a la concepción de ciencia que distanciaba a Durkheim de sus colegas filósofos de la tendencia anterior, como a su interés de subsumir la Ciencia de la Educación en los dominios de la Sociología. Así, la consideración de la pedagogía como teoría práctica constituye el reconocimiento de su carácter de reflexión dirigida a la acción. Y aquí es preciso tener en cuenta que Durkheim niega el carácter de arte para la pedagogía, pues, según él, un arte es, fundamentalmente, un conjunto de costumbres, prácticas, habilidades organizadas; el arte de la educación es el saberhacer del educador, la experiencia del profesor. La pedagogía, por el contrario, es un conjunto de ideas relativas a esas prácticas, es un conjunto de teorías que no tienen como objeto expresar lo real, sino orientar la conducta. Es por eso que se puede ser un buen profesor sin conocer mucho de pedagogía o, por el contrario, ser un buen pedagogo careciendo de cualquier habilidad práctica para la educación.

Debido al carácter eminentemente social concedido a la educación, su estudio hacía parte de la Sociología, y de esa forma despojaba Durkheim a la pedagogía del objeto que había sido designado para ella - como Ciencia de la Educación- por la tendencia anterior propia de la pedagogía filosófica, ahora parte de los objetos de su ciencia sociológica. Sobre el objeto de la pedagogía se preguntaba el sociólogo: “¿qué es la pedagogía sino la reflexión aplicada, lo más metódicamente posible, a las cosas de la educación con miras a regular su desarrollo?" (Durkheim, 2003, p. 103). Con ese movimiento, la Sociología procuraba escalar un grado más en el camino de la cientificidad, al mismo tiempo que pretendía utilizar los privilegios que alcanzó la pedagogía en el estudio y dirección de los problemas de la educación, en una época en que la expansión de la instrucción pública era un asunto estratégico para los intereses del Estado.

Estas dos tendencias esbozadas hasta aquí (una, ligada a la filosofía espiritualista francesa que intentó constituir una Ciencia de la Educación, o pedagogía, como teoría-práctica dependiente particularmente de la psicología; otra, vinculada a la naciente sociología que estableció una diferencia entre Ciencia de la Educación - de carácter sociológico-y pedagogía, ni ciencia, ni arte, teoría-práctica destinada a orientar la conducta educativa), junto con los posteriores desarrollos de la Psicología Experimental del francés Alfred Binet y la psicopedagogía de los suizos Edouard Claparède y Pierre Bovet, posibilitaron la aparición y desarrollo, durante el siglo XX, de las llamadas Ciencias de la Educación ${ }^{12}$.

12 Rios (2008) concuerda con los franceses Develay (2001) y Gautherin (2002), en que las cátedras de pedagogía o ciencia de la educación, dictadas en las universidades francesas entre 1883 y 1914, no son un acontecimiento anunciador de las Ciencias de la Educación. Sobre el proceso particular de constitución de las Ciencias de la Educación, tanto en Suiza y Bélgica como en Francia, ver: Hofstetter (2002), Charlot (1995), Gautherin (2002). Para el caso colombiano, ver Rios (2008). 


\section{La Bildung o de la educación a través de la enseñanza: surgimiento de la Pädagogik o Ciencia de la Educación en Alemania}

Para comprender de manera general el significado de Bildung, concepto clave de la cultura pedagógica germánica, vale la pena retomar el análisis de Klafki (1987), según el cual son cuatro las características centrales de las teorías clásicas de la Bildung ${ }^{13}$. En primer lugar, la Bildung se expresa mediante los conceptos de autodeterminación, libertad, emancipación, autonomía, mayoría de edad, razón, auto-actividad y, en ese sentido, significaría capacitación para la autodeterminación racional, en donde la auto-actividad es la forma central de realización del proceso formativo. Sobre este aspecto, Kant (apud Klafki, 1987, p. 45) escribía: "Mejorarse a sí mismo, cultivarse a sí mismo [...] crear moralidad en sí, eso es lo que el hombre debe hacer".

En segundo lugar, la Bildung puede ser entendida como el desenvolvimiento del sujeto en el mundo objetivo universal; sin embargo, esto no debe ser entendido como una perspectiva meramente subjetivista o individualista, pues para las teorías clásicas de la Bildung, el sujeto logra alcanzar la racionalidad -como parte de un proceso de apropiación y discusión crítica de la cultura-, que no se reduce a un plan de estudios sino a una formación, no conclusiva, que abarca la totalidad de la vida. En otras palabras, si la condición de la formación es la existencia de una cultura humana anterior, el concepto de Bildung privilegia las producciones humanas, las ganancias civilizatorias en la satisfacción de las necesidades, el conocimiento sobre la naturaleza y sobre el mundo humano, las acciones políticas, los sistemas de normas y acciones éticas, las formas de vida social, los productos estéticos, etc. Aquí los conceptos centrales son: humanidad, hombre, humano, mundo, objetividad, universal.

En tercer lugar, la Bildung implica una relación 'dialéctica' entre la individualidad y la colectividad, es decir, la formación de la individualidad es la manera particular como se concretiza, en cada caso, la objetividad general (mundo, cultura), y tal concretización sólo es posible mediante la apropiación y discusión

13 Es importante resaltar el uso del plural por parte de Klafki, pues se trata de un análisis de las diversas perspectivas sobre la Bildung y no de una teoría particular. Sobre este aspecto, él mismo aclara que la determinación de esas cuatro características no constituye una reproducción, sino una interpretación comparativa y sinóptica de los conceptos de diversos autores. crítica de la objetividad general. Esto no significa una limitación sino, por el contrario, la condición previa para el desarrollo de la plenitud que en potencia encierra, en cada caso, lo general. En otras palabras, la formación implica una relación dialéctica entre la capacidad de autodeterminación y un contenido objetivo general previo. Además, es necesario entender aquí la individualidad no sólo como referida a un sujeto específico: las naciones, los pueblos y las culturas son consideradas - dentro de las teorías clásicas de la 'formación' - como 'individualidades colectivas', es decir, como expresiones inconfundibles de humanidad posible dentro de un proceso histórico. De ahí la importancia formativa de la filosofía de la historia (Herder, por ejemplo), en cuanto se preguntaba por los avances, limitaciones y errores de esas distintas individualidades colectivas; de ahí, también, la idealización que los neo-humanistas alemanes hicieran de los griegos como modelo normativo, como ejemplo de humanidad no repetible, expresada en la famosa consigna bilde dich griegisch (fórmate como un griego).

En cuarto lugar, señala Klafki, el acuerdo en las teorías clásicas sobre la existencia de por lo menos tres dimensiones de la actividad humana (correspondientes a la división básica de la filosofía kantiana), que deben ser objetivo de la Bildung: la dimensión moral, la cognitiva y la estética. En el caso de Humboltd, se trata de la 'formación general del hombre'; para Pestalozzi, esas dimensiones corresponderían a la triple formación de la 'cabeza, el corazón y la mano', y en el caso de Herbart, se trataría de la formación de un 'interés múltiple' o multiplicidad del interés. La dimensión moral es considerada, en las teorías clásicas de la 'formación', un despertar de la responsabilidad moral auto-determinada, de la disposición para la acción moral y de la facultad de acción. En relación con la dimensión intelectual, del conocimiento o del pensamiento, las teorías clásicas, al decir de Klafki, jamás fomentan la racionalidad instrumental sin vínculo con la reflexión racional sobre el sentido humano, sobre la responsabilidad de sus posibilidades de aplicación. En este sentido, para comprender mejor lo que ello significa, resulta útil la diferenciación establecida par Kant entre intelecto (Verstand) y razón (Vernunft):

"Intelecto" designa, en sentido estricto de la palabra, la racionalidad instrumental por medio de la cual, en un proceso que no se puede concluir, se producen saber y conocimiento que luego pueden encontrar aplicación técnica, en principio para cualquier finalidad; "razón", por el contrario, se refiere a ese modo reflexivo de racionalidad según la cual nos preguntamos, por un lado, por los requisitos de los conocimientos fundados en el intelecto y, por otro, 
por la fundamentación de las metas perseguidas con la aplicación del saber y de los conocimiento; en el contexto de las teoría clásicas de la educación [Bildung] eso significa lo siguiente: preguntarse por las posibilidades y límites de la racionalidad instrumental para una existencia humana del hombre (Klafki, 1987, p. 55).

Según los análisis de Klafki, el énfasis que durante el siglo XIX tuvo esa dimensión cognitiva constituye uno de los aspectos de la decadencia del concepto de Bildung, en la medida en que borró el valor de, por ejemplo, la dimensión estética, cuya amplia extensión en las teorías clásicas abarcó conceptos como formación de la sensibilidad -en el sentido de perfeccionamiento de la facultad sensitiva-, frente a los fenómenos de la naturaleza y de la expresión humana; desarrollo de la facultad imaginativa o fantasía; desarrollo del gusto y de la facultad de gozo y juicio estéticos; capacidad para el juego y para la sociabilidad. La formación estética, en ese sentido, no era entendida como formación en la gran literatura, teatro, música o artes plásticas, sino en la idea de una estética de lo cotidiano, es decir:

[...] desde la bisutería al diseño de muebles, desde el vestido a la música popular; comprende igualmente el baile, los juegos, las fiestas, abarcando todas las formas de trato entre los hombres y todos los estilos de la sociabilidad, incluyendo también el erotismo - Schiller, por ejemplo - como el cultivo estético de la sexualidad natural. Una vez más podemos apreciar que los enfoques teóricos de aquella época sobre la educación van mucho más allá del estrecho marco de la educación escolar (Klafki, 1987, p. 57).

Schiller fue quien dio mayor importancia al problema estético en la teoría de la formación, y su filosofía fue un intento de solucionar el problema dejado por la estética kantiana ${ }^{14}$. Sin embargo, su concepción de la experiencia estética fue ambigua en la medida en que era considerada tanto un medio como un fin de la educación: medio o hilo metódico en el camino de la educación de la facultad racional político-moral del hombre; fin en cuanto posibilidad de la felicidad, de la satisfacción humana y de la idea de plenitud y esperanza de una buena vida futura por realizarse (Cfr. Klafki, 1987).

14 “... la filosofía kantiana para Schiller parece carecer de una conclusión: la Crítica del juicio, que estremeciera toda la estética de entonces, no lograría elevarla a la condición de doctrina del gusto. La estética kantiana parece haber permanecido en una mera 'propedéutica' — a la manera que 'preparó los fundamentos'- a la teoría del arte, y coincidiendo en este punto con toda la filosofía postkantiana, Schiller se propone como tarea completar el sistema previsto por Kant" (Suzuki, 2002, p. 8).

\section{Herbart y la nueva Ciencia de la Educación o Pädagogik}

La riqueza del saber pedagógico en los comienzos del siglo XIX encuentra su expresión más depurada en Herbart, cuyo trabajo es reconocido por algunos historiadores clásicos de la educación como el fundador de la "pedagogía moderna", o como la primera tentativa de una "pedagogía científica" (Hubert, 1976; Eby, 1976; Buisson, 1911; Compayré, 1906). Su propuesta de una educación a través de la enseñanza, expresada en su "Pedagogía General derivada del fin de la educación" (1806), es producto de una reelaboración, entre otras, de las ideas de Rousseau y de Kant (de las cuales se distancia en varios aspectos), sobre la base de la tradición didáctica del siglo XVII y de las teorías de la Bildung.

\section{"La riqueza del saber pedagógico en los comienzos del siglo XIX encuentra su expresión más depurada en Herbart, cuyo trabajo es reconocido por algunos historiadores clásicos de la educación como el fundador de la "pedagogía moderna", o como la primera tentativa de una "pedagogía cientifica"."}

Sin embargo, es preciso decir que el camino ya estaba abierto, de una parte, por las discusiones de los representantes franceses en la comisión creada por los revolucionarios para definir los rumbos de la instrucción pública, particularmente con el llamado "informe Condorcet" (Condorcet, 2001); de otra, por Kant, a propósito de sus elaboraciones sobre pedagogía recogidas en el texto redactado por su discípulo Theodoro Rink - Über Pädagogik o "Sobre la Pedagogía"-, con base en las notas de clase tomadas en las lecciones de pedagogía dictadas por el filósofo en sus cursos de 1776/77, 1783/84 y 1786/87, en la Universidad de Königsberg ${ }^{15}$.

15 Vale la pena mencionar aquí la dificultad de ciertos traductores en reconocer el papel de las particularidades culturales y lingüísticas en la comprensión de la riqueza conceptual del saber pedagógico. Es el caso de Lorenzo Luzuriaga quien en la edición española de la "Pedagogía" de Kant (2003), aclara lo siguiente en nota de pie de página: "Como ni en ésta ni en las restantes obras se encuentra una distinción precisa entre Bildung, formación y Erziehung, por educación, se ha traducido frecuentemente Bildung por educación, a fin de evitar confusiones" (p. 29). 
En su propuesta de una "educación a través de la enseñanza”, Herbart destaca dos aspectos: de una parte, la importancia de un conocimiento especializado del educador y, de otra, la relación estrecha de la educación y la enseñanza con la formación del carácter. Sobre el primer aspecto, Herbart inicia su libro cuestionando la ausencia de una opinión formada, en la mayor parte de los educadores, sobre lo que es la educación. Rousseau, dice el pedagogo alemán, por lo menos definió un punto de vista y permaneció fiel a él; siguió la naturaleza y buscó garantizar un desarrollo libre y alegre de todas las manifestaciones de la vida vegetativa humana, desde el nacimiento hasta el matrimonio. Pero una educación de ese género "es demasiado dispendiosa", pues el educador debe sacrificar su vida para acompañar al joven. Además, "criar hombres naturales significa, por ventura, repetir de nuevo todos los errores ya superados" (Herbart, 2003, p. 11).

No basta, entonces, seguir la naturaleza, pero tampoco es suficiente, para educar, tener experiencia, experimentar, pues "un maestro de escuela de aldea nonagenario tiene la experiencia de noventa años de vida rutinaria, tiene el sentido de su largo esfuerzo pero ¿será que también tiene el sentido crítico de sus resultados y de su método? (p. 11). Tenemos así que para el educador, en la concepción de Herbart, no basta la experiencia ni un saber superficial; debe poseer una ciencia como condición para la realización adecuada de su actividad educadora. Y esa ciencia es, justamente, la pedagogía. Ésta, a su vez, depende de la filosofía práctica y de la psicología: “aquella muestra el fin de la educación; ésta, el camino, los medios y obstáculos" (Herbart, s/d, p. 9). Pero no es suficiente sólo con la pedagogía: "la Pedagogía es la ciencia que el educador necesita para sí mismo. Sin embargo, él también tiene que poseer conocimientos que le permitan comunicar" (Herbart, 2003, p. 16). Es decir, necesita conocer la pedagogía para enseñar y la ciencia que va a enseñar o comunicar. De esta forma, el educador herbartiano se diferencia del antiguo maestro de escuela en el hecho de necesitar de la ciencia, de conocimientos sólidos y profundos, no sólo de un método general para desempeñar su tarea educativa.

Sobre el segundo aspecto - la relación de la educación y la enseñanza con la formación del carácter-Herbart establece una clara diferencia entre Erziehung (educación) y Unterricht (enseñanza); sin embargo, reconoce que una no puede estar separada de la otra: "Desde ya confieso no concebir la educación sin enseñanza, así como ya referí previamente, por lo menos en este escrito, que no concibo una enseñanza que no eduque simultáneamente" (2003, p. 16). La enseñanza (o instrucción) ocupa un lugar destacado en la pedagogía de Herbart, en la medida en que sin ella el trabajo educativo no podría alcanzar su fin. En otras palabras, la enseñanza es la clave de la formación del carácter y esto porque la enseñanza determina en gran medida los contenidos mentales; entonces, "es fundamental para el educador saber cómo se determina su modo de pensar [del alumno], una vez que es a partir del modo de pensar que se forman los sentimientos y, en función de éstos, principios y formas de conducta" (p. 16). El gobierno pedagógico es así un gobierno de los contenidos mentales que definirán la dirección de la conducta y, por tanto, garantizarán la virtud.

Según el pedagogo alemán existen dos fuentes iniciales de conocimiento: la experiencia y el trato social o convivencia. La primera hace referencia a la actividad del individuo sobre las cosas, sobre aquello que lo rodea, sobre el mundo; la segunda se refiere a aquella actividad donde lo fundamental es la interacción de los individuos. Ambas actividades ofrecen al individuo un conjunto importante de representaciones a partir de las cuales, y mediante un proceso de expansión, se va configurando el conocimiento. Pero, según Herbart, esas dos fuentes iniciales no son suficientes: es preciso que sean complementadas y, en cierta medida, organizadas y, aún, aumentadas mediante el proceso de enseñanza. Sólo ésta puede articular de forma coherente y ampliar el "círculo de ideas" (representaciones, percepciones) con los datos aportados por la experiencia y la convivencia, de manera que constituye la fuente más importante del conocimiento.

Por otro lado, la enseñanza tiene como fin inmediato la producción del "interés múltiple” y, como fin mediato, la virtud. Aunque la experiencia y el trato social contribuyen en la construcción de las representaciones y, de esa forma, permiten la ampliación del círculo de ideas, tal contribución es desordenada, dispersa y no tiene un objetivo claro presente. Por el contrario, sólo la enseñanza —en la medida en que debe ser sistemática- garantiza la integración adecuada, no sólo de las representaciones provenientes de la experiencia y la convivencia, sino de las representaciones de las ciencias que ella misma presenta de forma ordenada y según un plan cuyo horizonte es la formación del carácter del alumno. No es posible que el individuo conforme su carácter en la dirección de la moralidad sin el auxilio de la enseñanza, lo que significa la necesidad de intervención de ese otro agente que es el maestro o profesor.

Restaría por analizar el lugar de la "formación" (Bildung) en la pedagogía de Herbart. Hasta aquí no se ha mencionado ese concepto, principalmente 
porque en los dos textos estudiados no se encuentra un apartado específico dedicado al tema, como sí sucede con los conceptos de educación y enseñanza. Pero el hecho de no ocupar un lugar explícito en sus dos libros pedagógicos más importantes no quiere decir que tal concepto sea ignorado o menos relevante en sus elaboraciones pedagógicas. Siguiendo la línea de Kant, podríamos decir que para Herbart la formación (Bildung) está compuesta por la disciplina y la enseñanza ${ }^{16}$ : sin embargo, debemos recordar que, mientras para Kant la instrucción es la parte positiva de la educación y la disciplina es fundamentalmente negativa - coacción mecánica o "acción por la que se borra al hombre la animalidad” (Kant, 2003, p. 30)-, para Herbart la parte negativa es lo que se denomina "el gobierno de los niños" y, por el contrario, la disciplina (por su proximidad con la instrucción) es un aspecto educativo, es decir, positivo, cuyo fin es la formación del carácter.

Diría, entonces, que para Herbart la formación (Bildung) es la "educación a través de la enseñanza". Fórmula muy importante para comprender la cultura pedagógica germánica pues permite diferenciar los conceptos de educación, enseñanza y formación con base en su articulación. La formación no puede confundirse con la enseñanza, aunque comparta con ella el horizonte de la cultura (Willmann, 1948); la formación sería, entonces, una "enseñanza educativa" y obedece a una condición específicamente humana. Su Bosquejo para un curso de Pedagogía inicia con esta frase:

El concepto de formabilidad ${ }^{17}$ tiene un alcance mucho más amplio. Se extiende hasta para los elementos de la materia. Empíricamente puede ser seguido hasta en los elementos en el metabolismo de los cuerpos orgánicos. De la formabilidad volitiva se encuentran vestigios en el alma de los animales. Pero formabilidad de la voluntad para la moralidad, sólo reconocemos en el hombre. (Herbart, 1835, p. 1).

Decir que sólo el hombre es formable para la moralidad es decir que sólo él puede alcanzar la moralidad a través de la enseñanza (de la cultura).

16 Dice Kant (1803, [458]-[459]): “La Bildung comprende la disciplina y la instrucción” [Bildung begreift unter sich Zucht und Unterweisung]. Sin embargo, la conocida traducción de Luzuriaga (Akal, 2003), traduce ésta frase así: “La educación comprende la disciplina y la instrucción" (p. 31), confundiendo Bildung con educación. Para Kant la educación - Erzeihunges más amplia que la formación —Bildung —, pues aquella comprende el cuidado - Wartung - y la formación, es decir, el cuidado junto con la disciplina y la instrucción.

17 El traductor español del texto de Herbart utiliza la expresión "educabilidad", pero la palabra empleada por el autor alemán es Bildsamkeit, derivada de Bilden (formar) y, por tanto, debería traducirse como "formabilidad".
Formabilidad sería, entonces, la capacidad humana de adquirir una determinada forma moral (formación del carácter) mediante la enseñanza. De este modo, la pedagogía de Herbart retoma la tradición didáctica y mediante la Bildung actualiza y justifica la instrucción o la enseñanza (y con ella, el papel y lugar del maestro), vinculándola a los desarrollos de la educación. Y fue esa respuesta de Herbart a la educación de Rousseau la marca particular de la nueva pedagogía moderna, marca que a finales del siglo XIX es cuestionada duramente por los desarrollos de la psicopedagogía francófona y la psicología educacional angloamericana, las cuales, sobre la base del concepto de aprendizaje, actualizarán la educación rousseauniana y su énfasis en el sujeto que aprende, constituyendo todo una "economía de la enseñanza" y abriendo, así, la posibilidad para la educación permanente a lo largo de toda la vida, es decir, para la ciudad educativa o la sociedad del aprendizaje.

\section{La Teoría del Currículo o los Curriculum Studies}

La cultura pedagógica angloamericana de los Curriculum Studies es la más reciente de las tradiciones intelectuales y, sin embargo, es la más influyente y extendida. Su consolidación y desarrollo se dio a partir de las primeras décadas del siglo XX, particularmente en los Estados Unidos de América y su propagación más allá del mundo anglófono fue producto, fundamentalmente, del movimiento de "mundialización de la educación" generado a fines de la década de 1960. Como consecuencia de esa expansión o "explosión" del currículo (Terigi, 1996) se llegó, durante la década de 1990, tanto en el caso angloamericano como iberoamericano, "a un estado en el cual todo lo que acontece en la institución escolar y en el sistema educativo es, de manera indiferenciada, curriculum" (Terigi, 1991, p. 161).

La fuerza de la expansión del campo de los estudios curriculares fue tal, que el currículo aparece como la forma propia en que se desarrollaron las prácticas educativas en la cultura occidental, es decir, aparece como la única forma, por tanto, de validez universal que asumió la organización de la educación desde épocas remotas. Para analizar la emergencia de esa cultura pedagógica se utiliza aquí el esbozo de "genealogía del curriculum escolar" elaborada por Terigi (1996), hace poco más de una década. El argumento central del análisis es el siguiente: "Que 'siempre' hayan existido ideas sobre lo que se debe enseñar no significa que 'siempre' haya existido el curriculum" (Terigi, 1996, p. 164). Sobre la base de 
esta afirmación, la investigadora, siguiendo una perspectiva foucaultiana, se opone a la tesis de un origen contraponiendo la idea de emergencia: "El origen remite a un punto mítico en que algo comienza a existir, aparta sus ocurrencias anteriores y supone su identidad subsecuente. La emergencia, al contrario, es 'la entrada en escena de las fuerzas; es su irrupción, el movimiento brusco por el cual ellas saltan de los bastidores a la escena'" (p. 170).

La perspectiva genealógica de Terigi intenta, entonces, evitar los problemas de anacronismo (considerar el currículo como presente a lo largo de toda la historia de la educación occidental), nominalismo (considerar que la aparición del término marca su origen) y contextualismo (restringuir su aparición al contexto de los Estados Unidos de América), presentes en las perspectivas estudiadas por ella y, para eso, emprende un análisis de las múltiples ocurrencias del término curriculum "no es todas las ocurrencias - eso es imposible-pero sí en la mayor variedad posible" (p. 170). La búsqueda de los diversos sentidos específicos se orienta en la perspectiva del curriculum como "prescripción acerca de la enseñanza, y más específicamente de los contenidos de enseñanza" (p. 171), pues, a pesar de la gran disparidad de definiciones, esa comprensión está presente en buena parte de las conceptualizaciones de los teóricos del campo.

En un sentido más específico, la idea de prescripción sobre la enseñanza se entiende, siguiendo los análisis de Hamilton (1992), como "una cierta secuencia, un determinado orden que produce el curriculum como plan de estudios" (Citado por Terigi, 1996, p. 171). De ahí que esta autora, al contrario de Hamilton (1992), considere que antes de la aparición del término en la Universidad de Glasgow, el Ratio Studiorum de los jesuitas incorporaba ya unas prescripciones sobre los contenidos a ser enseñados basados en una perspectiva de secuencia y orden; por eso, "una historia del curriculum que comenzase apenas en Glasgow, dejaría el Ratio Studiorum por fuera” (p. 167).

Continuando en la búsqueda de los sentidos del curriculum, Terigi menciona como otro momento importante en su desarrollo la constitución de los
Estados Nacionales Europeos, la nueva categoría de ciudadano y los esfuerzos de las administraciones estatales para definir una institución educativa específica (la escuela). En particular, señala cómo la constitución de la escuela nacional estuvo íntimamente vinculada a la definición de los planes educativos nacionales y, principalmente, destaca el caso francés del Informe sobre la instrucción Pública presentado por Condorcet a la Asamblea Nacional Legislativa en abril de 1792. Tal informe "contiene una propuesta de graduación de la enseñanza, una previsión de los destinatarios y docentes para cada grado de instrucción y una indicación expresa de los contenidos a enseñar en cada uno" (p. 172); por tal motivo, forman parte de la historia del currículo. Siguiendo esa lógica, Terigi concluye que la producción curricular estadunidense sólo incrementa un sentido específico al currículo: "ya no se trata de construir una propuesta de estudios destinada a construir el ciudadano, sino de construir el sujeto característico de una sociedad industrial" (p. 173).

La presentación detallada de la perspectiva de la autora, obedece a una razón metodológica: argumentar la consideración de la emergencia, a mediados del siglo XIX, de la perspectiva curricular como una tradición específicamente anglosajona $y$, por eso, diferente de la pedagogía y la didáctica germánica, así como de la ciencia de la educación francesa. Y, como se dijo aquí, en la presentación de las tradiciones, diferente no significa ni completamente aislada ni perteneciente a otra formación discursiva. Diferente quiere decir que no es idéntica, que no se trata de una simple substitución de términos de una lengua por los de otra, aunque las tres tradiciones compartan un mismo suelo, arqueológicamente hablando, en tanto constituyen modificaciones, alteraciones, apropiaciones particulares producto de la cultura. En ese sentido, se diría que la genealogía de Terigi queda presa en la "explosión" que ella misma denuncia del campo del currículo; no consigue salir de la perspectiva curricular, no consigue percibir la existencia de las otras tradiciones intelectuales, pues las diferencias encontradas en su revisión histórica son sólo variantes del currículo que, a su vez, es confundido con la didáctica. 
Es evidente que el currículo comparte con la didáctica la "prescripción de la enseñanza" pero en ese caso se diría que, como la constitución de la didáctica es muy anterior a la perspectiva del currículo, éste último retoma ese aspecto de la didáctica. Aquello que identifica la perspectiva curricular no es la prescripción de los contenidos de la enseñanza, sino la organización de la enseñanza como un conjunto de actividades o experiencias organizadas, según las actividades y experiencias que se espera que los niños desarrollen en su vida adulta. Esta no es una característica compartida ni con la Didáctica, ni con la Ciencia de la Educación francófona. Se trata de una perspectiva anclada en el utilitarismo y en el pragmatismo de procedencia anglosajona, como pasaremos a analizar a continuación.

Herbert Spencer (1820-1903), abre su libro Educación física, intelectual y moral (1860) con la siguiente cuestión: “¿Cuáles son los conocimientos más útiles?", dejando en claro de entrada su perspectiva. Su tesis inicial es la de que, en la historia de la humanidad, se puede reconocer fácilmente el privilegio dado a las cosas agradables frente a las útiles, tanto en el plano intelectual como en el material. Una de las pruebas de su afirmación está en el hecho de que, por ejemplo, los primitivos habitantes de América prefirieron el adorno (plumas, tinturas, aretes, etc.) al vestido, lo que tiene su explicación en otro hecho: la subordinación, desde tiempos remotos, de las necesidades individuales a las sociales, es decir, el imperio de la sociedad sobre el individuo (Spencer, 1860).

Para revertir esa tendencia, es preciso tener en cuenta cuáles son las actividades útiles para la vida humana y clasificarlas según su importancia. Spencer define cinco de ellas: 1) Actividades que contribuyen directamente a la conservación del individuo; 2) Actividades que contribuyen indirectamente en ella; 3) Actividades empleadas en educar y disciplinar la familia; 4) Actividades que garantizan la manutención del orden social y de las relaciones políticas y, 5) Actividades para ocupar los momentos de ocio y descanso (Spencer, 1860). La educación ideal sería la preparación completa para la posesión de todas las actividades, aunque sólo será posible, en la realidad, mantener un grado razonable de preparación para ellas.

Según esa lógica, "son estas ideas generales con las cuales tenemos que discutir un curriculum: división de toda la vida en actividades de géneros diferentes y de importancia creciente; el valor de cada orden de hechos, según sea el tipo de actividad connatural, semi-convencional o convencional; doble influencia de ellas en cuanto conocimiento y disciplina" (Spen- cer, 1860, p. 19). De esta forma Spencer deja en claro lo que es el currículo, en tanto perspectiva particular de pensamiento y organización de la educación, perspectiva que se encontrará décadas después, en Bobbitt $(1918,1924)$, Thorndike (1936) y hasta en Tyler (1974 [1949]).

Con Spencer, aparece entonces, por primera vez, una definición de currículo que va más allá de la noción de "plan de estudios" o de enseñanza, para abarcar el sentido mismo de la educación como práctica organizada "racionalmente" sobre el principio de la utilidad. De ahí que si, bajo la consideración de que el fin de la educación es "preparar nos para la vida completa" la pregunta esencial debe ser: “¿cómo se debe vivir?". Esa cuestión esencial y sus preguntas derivadas constituyen la "manera racional de juzgar un recorrido educacional y de saber en qué grado consigue ese fin" (Spencer, 1860, p. 12). Podría parecer que la definición de un orden racional para el "recorrido educacional" fuese también el asunto de la Ratio Studiorum de los jesuitas y hasta de la propia Didáctica Magna de Comenio; por tanto, no existirían diferencias significativas entre aquellas formas y el curriculum anglosajón. Terigi acertaría al considerar que ellas formarían parte de la historia del currículo. Sin embargo, aquí se reafirma la perspectiva de diferenciar entre aquella vía didáctica y el curriculum, aunque sea evidente que no se trata de algo completamente diferente.

La vía curricular, digamos así, es la vía utilitarista que, pensando la educación en función de su utilidad para el individuo y para la sociedad, la organiza sobre la base de una clasificación de las actividades constitutivas de la vida humana según su importancia su valor (connatural o convencional) y su influencia en los conocimientos y en la disciplina. Todo eso en función de garantizar que el individuo pueda vivir una "vida completa". No es una clasificación de los saberes, de los conocimientos, de las materias, de los contenidos teóricos que deber ser enseñados, sino de las "actividades" (más tarde Bobbitt, Thorndike y Tyler hablarán de "experiencias") de la vida en el momento particular de la sociedad en la que se encuentra el individuo. Debido a su fundamento en las actividades, la concepción de educación de Spencer también resulta diferente de la línea didáctica, pues antes que "erudición" y, por tanto, de un énfasis en la enseñanza, se trata de una recurrencia a la capacidad de actuar (agencia) y a la posibilidad de autoregulación del individuo que aprende. Esos elementos fundamentales del currículum, que aparecen por primera vez formulados en el texto de Spencer, van a ser retomados y desarrollados a comienzos del siglo XX, 
principalmente en el contexto norteamericano, por autores como Franklin Bobbitt e Edward Thorndike, entro otros, constituyendo lo que Kliebard (1979), llamó "campo del currículo"18.

Es esa tentativa de definir los fines de la educación a partir de la clasificación de las actividades de la vida humana adulta, esa preocupación con la experiencia, con la actividad del individuo que aprende, esa preocupación por la utilidad de esas actividades y de los conocimientos que deben ser enseñados y aprendidos, aquello que diferencia la cultura pedagógica de los Curriculum Studies de las otras dos tra-

18 Sobre la historia de la constitución del "campo del currículo" en los Estados Unidos, su relación con el problema del control social y con los desarrollos del Scientific Management de F. W. Taylor, ver: Apple (1979), Kliebard (2004).

\section{Bibliografía}

Academie Française. (1694), Dictionnaire de l'Académie française. Recuperado el 7 de febrero de 2009 de: http://artflx.uchicago.edu/

Apple, M. (1979), Ideology and curriculum. London: Routhledge and Kegan Paul.

Bobbitt, F. (1924), How make a curriculum. Boston: Houghton Mifflin.

Bobbitt, F. (1918), The Curriculum. New York: Houghton Mifflin Company.

Buisson, F. (1911), Nouveau Dictionnaire de pédagogie et D 'Instruction Primaire. Paris: Librairie Hachette. Recuperado el 20 jun. 2008, de http://www.inrp.fr/editionelectronique/lodel/dictionnaire-ferdinand-buisson/.

Charbonnel, N. (1988), Pour une critique de la raison éducative. Berne: Peter Lang.

Charlot, B. (1995), Les sciences de l'éducation, un enjeu, un défi. Paris : ESF, Editeur.

Claparède, E. (2007), La educación funcional. Traducción de Mercedes Rodrigo. Madrid: Biblioteca Nueva.

Compayré, G. (1897), Cours de pédagogie theorique et practique. Paris: Librairie Classique Paul Delaplane.

(1906), Herbart et l'éducation par l'instruction. Paris: Delaplane.

(1920), Curso de Pedagogía. México: Librería de la Vda. de Ch. Bouret.

Condorcet. (2001), Cinco memorias sobre la instrucción pública y otros escritos. Madrid: Morata.

Coombs, Ph. (1971), La crisis mundial de la educación. Barcelona: Península. diciones formadas de manera más o menos paralela. Sin embargo, es preciso dejar una cosa en claro: la vía del curriculum no agota la producción anglosajona, pues ella es más diversa y rica como lo han mostrado los estudios sobre la obra de John Dewey, Granville Stanlye Hall o E. Thorndike, por ejemplo. Lo que aquí se quiso mostrar no fue el hilo común a toda la producción pedagógica de la cultura anglosajona; la intención fue mostrar sólo una parte, aquella que constituyó lo más particular, lo sui generis y, por tanto, aquello que permite establecer una clara diferencia entre ésta y las otras dos culturas pedagógicas que se constituyeron en la Modernidad.

Corominas, J. (1954), Diccionario crítico etimológico de la lengua castellana. Madrid: Gredos.

Cunha, A. (1986), Dicionário etimológico Nova Fronteira da Língua Portuguesa. Rio de Janeiro: Nova Fronteira.

Develay, M. (2001), Propos sur les sciences de l'éducation, Réflexions épistémologiques. Paris : ESF.

Dilthey, G. (1968), Historia de la Pedagogía. Buenos Aires: Losada.

Dubois, P. Configurations et figurations d'une «science de l'éducation» dans le Dictionnaire Pédagogie de Ferdinand Buisson. In: Symposium «Science(s) de l'éducation et République face à face. Thórisations contrastées d'une discipline indisciplinée (fin du 19e-20e)», (2007), Stransbourg. Recuperado en Julio de 2009 de: http:// www.congresintaref.org/actes_pdf/AREF2007_Patrick_DUBOIS_341.pdf.

Durkheim, E. (2002), La educación moral. Madrid: Morata.

Durkheim, E. (2003), Educación y Sociología. Barcelona: Península.

Eby, F. (1976), História da Educação Moderna. Teoria, organização e práticas educacionais. Porto Alegre: Globo.

Estienne, R. (1552), Dictionarium latinogallicum. Recuperado el 20 de agosto de 2009 de: http://artfl-project. uchicago.edu/node/65

Foucault, M. (2006), Seguridad, Territorio, Población. Buenos Aires : Fondo de Cultura Económica.

Foucault, M. (2007), Nacimiento de la biopolítica. Buenos Aires : Fondo de Cultura Económica. 
Gautherin, J. (1995), La science de l'éducation, discipline singulière: 1883-1914. In Charlot, Bernard. Les sciences de l'éducation, un enjeu, un défi. Paris: ESF éditeur.

Gautherin, J. (2002), Une discipline pour la République: La Science de l'éducation en France (1882-1914). Berna: Peter Lang.

Hamilton, D. (1992), Sobre as origens dos termos classe e curriculum. Teoria \& Educação 6, 33-52.

Hameline D. (2002), Le statut de la pédagogie. Encyclopaedia Universalis, XVII.590-593.

Herbart, J. F. (s/d), Pedagogía General. Madrid: La Lectura. (1835), Umriss pädagogischer Vorlesungen. Göttingen.

(2003), Pedagogia Geral. Lisboa: Fundação Calouste Gulbenkian.

Hofstetter, R., \& Schneuwly, B. (Eds.) (2002), Le pari des sciences de l'éducation. Bruxelles: Éditions De Boeck Université.

Hopmann, S., \& Riquarts, K. (2000), Starting a Dialogue: A beginning conversation between didactik and the curriculum traditions. In: Westbury, I., Hopmann, S., \& Riquarts, K. (Eds.). Teaching as a Reflective Practice. New Jersey: Lawrence Erlbaum Associates Inc.

Houssaye, J., et al. (2004), Manifesto a favor dos pedagogos. Porto Alegre: Artmed.

Hubert, r. (1952), Tratado de Pedagogía General. Buenos Aires: El Ateneo.

Hubert, R. (1976), História da Pedagogia. São Paulo: Companhia Editora Nacional.

Kant, I. (1803), Uber Pädagogik. Recuperado el 6 de agosto de 2009 de: http://de.wikisource.org/wiki/ Über_Pädagogik\#fertig

Kant, I. (2003), Pedagogía. Madrid: Akal.

Kemmis, S. (1988), El curriculum, más allá de la teoría de la reproducción. Madrid: Morata.

Klafki, W. (1987), La importancia de las teorías clásicas de la educación para una concepción de la educación general hoy. Educación, Núm.36, pp. 40-65.

Kliebard, H. (1979), The Drive for Curriculum Change in the United States, 1890-1958. I - The Ideological Roots of Curriculum as a Field of Specialization. Curriculum Studies, Núm. 11 (3), pp. 191-202.

Kliebard, H. (2004), The struggle for the American Curriculum. 1893-1958. New York: RouthledgeFalmer.
Lenzen, D. (1996), La Ciencia de la Educación en Alemania: Teorías, crisis, situación actual. Educación, 54, 7-20.

Martínez, A., Noguera, C. \& Castro, J. (2003), Currículo y modernización. Cuatro décadas de educación en Colombia. Bogotá: Cooperativa Editorial Magisterio-Grupo Historia de las Prácticas Pedagógicas.

Melton, J. (2002), Absolutism and the eighteen-century origins of compulsory schooling in Prussia and Austria. First paperback edition. Cambridge: Cambridge University Press.

Narodowski, M. (1995), La pedagogía moderna em penumbras. Perspectivas históricas, en: Propuesta Educativa, Buenos Aires, Año 6, No. 13, p. 19-23.

Narodowski, M. (2001), Comenius e a Educação. Belo Horizonte: Autêntica.

Noguera, C. (2009), O governo pedagógico: Da Sociedade do Ensino para a Sociedade da Aprendizagem. Tese de Doutorado, Universidade Federal do Rio Grande do Sul, Porto Alegre, RS. Brasil.

Noguera, C. (2010), Governamentalidade e educação. A Modernidade como uma sociedade educativa. In: Neutzlin, Inácio. XI Simpósio Internacional IHU: O (des)Governo biopolítico da vida humana. Instituto Humanitas. Casa Leiria, São Leopoldo, RS, 2010. Disponible en: http:// www.ihu.unisinos.br/index.php?option=com_publicac oes\&Itemid $=20 \&$ task $=$ categorias \&id $=15$

Nóvoa, A. As ciências da educação e os processos de mudança. In: Nóvoa, A., Ponte, J., \& Bredero de Santos, M. (EDs.). (1991), Ciências da educação e mudança. Porto: Sociedade Portuguesa de Ciências da Educação.

Nóvoa, A. (1997), Regards nouveaux sur l'éducation nouvelle. In: Carbonnel, N. (Org.). Le don de la parole. Berne: Peter Lang.

Ramos do Ó, J. (2003), O governo de si mesmo. Modernidade pedagógica e encenações disciplinares do aluno liceal (último quartel do século XIX - meados do século XX). Lisboa: Educa.

Real Academia Española. (1992), Diccionario de la Lengua Española. Tomo I. Madrid: Espasa-Calpe, S.A.

Rios, R. (2008), Las ciencias de la educación en Colombia, 1926-1954: entre universalismo y particularismo cultural. Bogotá: Cooperativa Editorial Magisterio-GHPP.

Rousseau, J. J. (1984), Emilio o de la educación. México: Porrúa.

Rousseau, J.J. (1999), Emílio ou da educação. São Paulo: Martins Fontes. 
Schriewer, J. (1991), La construcción de la pedagogía científica. Diferenciación institucional y disciplinar, funciones formativas y praxis educativa de la ciencia universitaria de la educación en Alemania y Francia. Educación, 296, 137-174.

Spencer, H. (1860), Education: Intellectual, Moral, and Physical. New York and London: D. Appleton and Company.

Suzuki, M. (2002), o belo como imperativo. In: Schiller, F. A educação estética do homem. São Paulo: Editora Iluminuras, Ltda.

Terigi, F. (1996), Notas para uma genealogia do curriculum escolar. Educacão e Realidade, 21, (1), 159-186.

Thorndike, E. \& Gates, A. (1936), Princípios elementares de educação. São Paulo: Saraiva.

Tyler, R. (1974), Princípios básicos de currículo e ensino. Porto Alegre: Globo.
Westbury, I., Hopmann, S., \& Riquarts, K. (Eds.), (2000), Teaching as a reflective practice. The German Didaktik Tradition. New Jersey: Lawrence Erlbaum Associates, Inc.

Willmann, O. (1948), Teoría de la Formación Humana. Madrid: Instituto "San José de Calasanz" de Pedagogía.

Wulf, C. (1999), Introducción a la Ciencia de la Educación. Entre teoría y práctica. Medellín: Asonem-Universidad de Antioquia.

Zuluaga, O., Herrera, S. (2009), La configuración de campos conceptuales como posibilidad para estudiar las culturas pedagógicas, en: Martínez, A., Peña, F. (Comp.). Instancias y Estancias de la Pedagogía. La pedagogía en movimiento. Bogotá: Bonaventuriana. 